\title{
人脑乙酰胆碱酯酶的单克隆抗体 及其抗原决定簇
}

\author{
朱美财 孙曼雾 辛颜柇(1) 方允中(2) \\ (军事医学科学院毒物药物研究所、(1)微生物与流行病研究所、(2)故射医学研究所, 北京 100850)
}

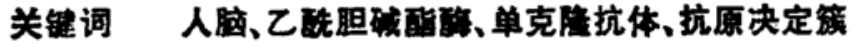

乙酰胆碱酯酶( AChE, EC, 3・1・1・7) 广泛分布于人神经组织, 还分布于人红细胞膜及 血清等非神经组织. AChE 即是分泌型蛋白质,也是膜结合蛋白质,一般定位于细胞膜或突触 前后膜, 但某些细胞的亚细胞结构中也含有 AChE. 已知在早老性痴呆患者脑组织某些区域 $\mathrm{AChE}$ 活性降低 ${ }^{[1]}$, 在先天性神经管缺损胎儿羊水中 $\mathrm{AChE}$ 活性增高 ${ }^{[2]}$, 但由于变性 $\mathrm{AChE}$. 的存在及可能的人红细胞膜 AChE 与假性胆碱酯酶的污染, 在免疫组织化学及滥床 AChE: 测定时需用脑 AChE 特异性抗体以便进行区别检定. 由于人脑 $A C h E$ 含量很低, 得到纯化 的 AChE 比较困难, 国外报道的抗人脑 $\mathrm{AChE}$ 单克隆抗体仅有三株 ${ }^{[3]}$, 并且与人红细胞膜 $A C h E$ 均有交叉免疫反应. 我们进行了人脑 $\mathrm{AChE}$ 单克隆抗体的制备工作, 找到了与人红细 胞膜 $A C h E$ 无交叉反应的单克隆抗体, 可能应用于神经系统 $\mathrm{AChE}$ 的免度组化研究或先天 性神经管缺损的产前诊断。

\section{1 材料与方法}

\section{1 抗人脑 $\mathrm{AChE}$ 单克隆抗体的制备 ${ }^{[019}$}

以 ConA 及短臂配基亲和层析纯化的人小脑 $\mathrm{AChE}$ (比活性 1300-1800U/mg 蛋白)为 抗原,与完全福氏佐剂混合后免度 $\mathrm{Balb} / \mathrm{c}$ 小鼠, 第一次每只腹腔注射 $50 \mu \mathrm{g}$, 再反复多次腹腔 注射, 每次每只 $30 \mu \mathrm{g}$. 于 $2-3$ 个月后取脾细胞与 SP2 $/ 0$ 骨艊瘁细胞融合, 䇥选阳性克隆. 经多次亚克隆后将阳性克隆培养增殖, 取适量杂交瘾细胞注射人 Balb/c 小鼠腹腔制备单克 隆抗体腹水。

\section{2 杂交部篇选及抗原抗体反应的检测}

采用间接 ELISA 法. 人脑 AChE 检测抗原为用 ConA 及两次配基亲和层析纯化的纹 状体及部分丘脑 AChE, 比活珄 $3000 \mathrm{U} / \mathrm{mg}$ 蛋白. SDS-PAGE 基本为一条主带 ${ }^{[0}$. 人红细胞 瞙 AChE 按文献 [6] 方法制备,比活性 $4000 \mathrm{U} / \mathrm{mg}$ 蛋白, SDS-PAGE 呈一条主带. 电鳐 AChE 按文献 [7] 方法制备, 比活性 7000U/mg 蛋白, SDS-PAGE 呈一条主带. 将抗原以包被液稀 释后, 每孔 $100 \mu \mathrm{l}$ 包被聚苯乙烯微量滴定板 $4^{\circ} \mathrm{C}$ 过夜. 洗涤后以 $1 \% \mathrm{BSA} 37^{\circ} \mathrm{C}$ 封闭 $1 \mathrm{~h}$, 加人 细胞培养上清液或不同稀释度的第一抗体 $37^{\circ} \mathrm{C}$ 娐温 $1 \mathrm{~h}$, 再加人辣根过氧化物酶标记的羊抗 鼠 IgG (1:1000 稀释) $37^{\circ} \mathrm{C}$ 反应 $1 \mathrm{~h}$. 然后加人底物邻苯二胺溶液室温暗化显色 $15 \mathrm{~min}$, 用

1992-07-08 收稿,1993-04-03 收改稿.

- 国家自然科学基金资助项目. 
$2 \mathrm{~mol} / \mathrm{L} \mathrm{H}_{2} \mathrm{SO}_{4}$ 终止反应, 于 492.1m 处测消光俧.

\section{3 变性人脑 $\mathrm{AChE}$ 及还原并烷化电熎电器官 $\mathrm{ACh}$ 抗原的制备}

纯化的天然人脑 $\mathrm{AChE}$ 于沸水浴中劣沸 $2 \mathrm{~min}$, 酶活性全部老失, 即热变性人脑 $\mathrm{AChE}$ 抗 原。纯化的电鳐电器官 $\mathrm{AChE}$ 的 $0.5 \mathrm{~mol} / \mathrm{L}$ Tris $-\mathrm{HCl}(\mathrm{pH} 8.2)-5 \mathrm{mmol} / \mathrm{L}$ EDTA 溶 液, 在 $6 \mathrm{~mol} / \mathrm{L}$ 盐酸胍存在下, 以终浓度 $20 \mathrm{mmol} / \mathrm{L}$ 二硫苏糖醇还原, 在 $37^{\circ} \mathrm{C}$ 通柔气搅拌 $2 \mathrm{~h}$, 然后加

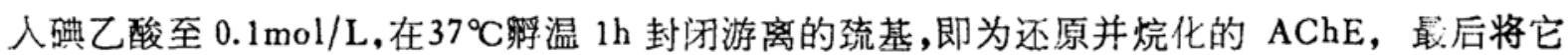
通过 Sephadex G-25 凝胶杜或对缓冲液适析 $48 \mathrm{~h}$ 除盐.

\section{4 还原并烷化的电媱电器官 AChE 鈢基侧链的破坏}

参照文献 [8] 进行, 以高碘酸篻化法裂解 $\mathrm{AChE}$ 分子的糖基侧链. 将还原并烷化的电鳐 电器官 AChE $(2 \mathrm{mg} / \mathrm{ml}) 1: 100$ 稀释后于 96 孔板包被过夜, 以洗涤液洗涤, 再以 $50 \mathrm{mmol} / \mathrm{L}$ 的乙酸钠缓冲液 ( $\mathrm{pH} 4.5)$ 洗涤一次. 加入 $20 \mathrm{mmol} / \mathrm{L}$ 高碘酸钠 $100 \mu 1$, 室温暗置 $1 \mathrm{~h}$. 留干, 用乙酸钠缓冲液冲洗三次. 加 $1 \%$ 甘氨酸 $100 \mu 1$, 室温封闭 $30 \mathrm{~min}$, 以洗浲液冲洗后加人 $1 \%$ ESA 封闭液封闭, 然后以间接 ELISA 法比较高碘酸氧化处理前后 AChE 抗原性的变化.

\section{5 抗人脑 $\mathrm{AChE}$ 单克隆沉体的亚类鉴定及纯化}

亚类鉴定采用免疫双扩散法,标准羊抗小鼠 Ig 亚类抗血清为 Sigma 公司产品。琼脂糖浓 渡为 $1 \%$,含 $3 \%$ PEG 6000 . 取浓缩 $30-40$ 倍的细胞上清液 $10 \mu 1$ 与标准抗血清 $10 \mu 1$. 在 $37^{\circ} \mathrm{C}$ 反应 $24 \mathrm{~h}$ 后观察沉淀线.单克隆抗体的纯化参照文献 [9]方法进行. 取腹水与等量生理盐水混 合后先以 $30 \%$ 饱和度的硫酸铵沉淀, 取上清液, 再以 $50 \%$ 饱和度的硫酸锫沉淀. 将沉淀物溶 于小体积的 $50 \mathrm{mmol} / \mathrm{L}$ 磷酸缓冲液 ( $\mathrm{pH} 8.0)-0.02 \% \mathrm{NaN}_{3}$ 中进行层析纯化. 预先以上述缓 冲液平衡 Sephadex G-200 柱, 样品上柱后以同样缓冲液洗脱, 按峰收集. 将第一峰对 $5 \mathrm{mmol} / \mathrm{L} \mathrm{Tris}-\mathrm{HCl}(\mathrm{pH} 8.5)$ 透析 $36 \mathrm{~h}$ 后, $10000 \times \mathrm{g}$ 离心, $30 \mathrm{~min}$ 取沉淀溶于 $0.1 \mathrm{~mol} / \mathrm{L}$ Tris $-\mathrm{HCl}(\mathrm{pH} 8.5)$ 缓冲液中. 以 SDS-PAGE 检定抗体纯度, 以 Lowry 法测定抗体浓度.

\subsection{AChE 活性测定}

参照微量羟胺比色法 ${ }^{[10]}$. 将纯化的人脑纹状体及部分丘脑 $\mathrm{AChE}(0.16 \mathrm{mg} / \mathrm{ml})$ 以 0.1 $\mathrm{mol} / \mathrm{L}$ 磷酸钠缓冲液 ( $\mathrm{pH} 7.4)-0.1 \%$ Triton X-100 按 $1: 100(\mathrm{~V} / \mathrm{V})$ 稀释后, 取不同量的缓 冲液补至 $50 \mu 1$, 加 $0.014 \mathrm{~mol} / \mathrm{L}$ 涣化乙酰胆碱 $50 \mu 1$, 在 $37^{\circ} \mathrm{C}$ 水浴中温育 $30 \mathrm{~min}$, 而后依次加入 碱性羟胺 $200 \mu 1,2 \mathrm{~mol} / \mathrm{L} \mathrm{HCl} 200 \mu 1$ 及 $0.197 \mathrm{~mol} / \mathrm{L} \mathrm{FeCl}_{3} 300 \mu 1$ 终止反应并呈色, $\mathrm{AChE}$ 的最低检测浓度约 $30 \mathrm{ng} / \mathrm{ml}$. 以微量多道扫描仪在 $492 \mathrm{~nm}$ 波长比色, 计算 $\mathrm{AChE}$ 活性. 测 定抗体对酶活性抑制作用时, 取 AChE 适量加人不同浓度的纯化单克隆抗体, $37^{\circ} \mathrm{C}$ 反应 $1 \mathrm{~h}$ 后再以上述方法测定酶活性.

\section{2 实 验 结 果}

\section{1 抗人脑 $\mathrm{AChE}$ 单克隆抗体的制备}

获得了三个稳定地分泌抗人脑 $A C h E$ 单克隆抗体的杂交瘤细胞株 $B_{10}, H_{11}$ 及 $E_{11}$. 当 每孔包被人脑 $\mathrm{AChE} 0.16 \mu \mathrm{g}$ 时, 以间接 ELISA 法测得腹水滴度分 别 为 96000,48000 及 24000. 三个单克隆抗体在高浓度情况下表现出过量抑制现象（前带效应）。亚类检定显示 $B_{10}, H_{11}$ 及 $E_{11}$ 三个单克隆抗体均为 IgM. 以凝胶过滤法纯化的三个单克隆抗体的产率分别 为每毫升腹水 $0.8,1.9$ 及 $1.54 \mathrm{mg}$ 抗体. 纯化的抗体经 ELISA 测定滴 度 在 1000-5000之 间. SDS-PAGE 在还原状态下均显示为重、轻链两条主带,分子量约 69 及 $22 \mathrm{kD}$ (图 1). I 
3-15\% SDS梯度胶电泳不还原状态下呈单一区带。

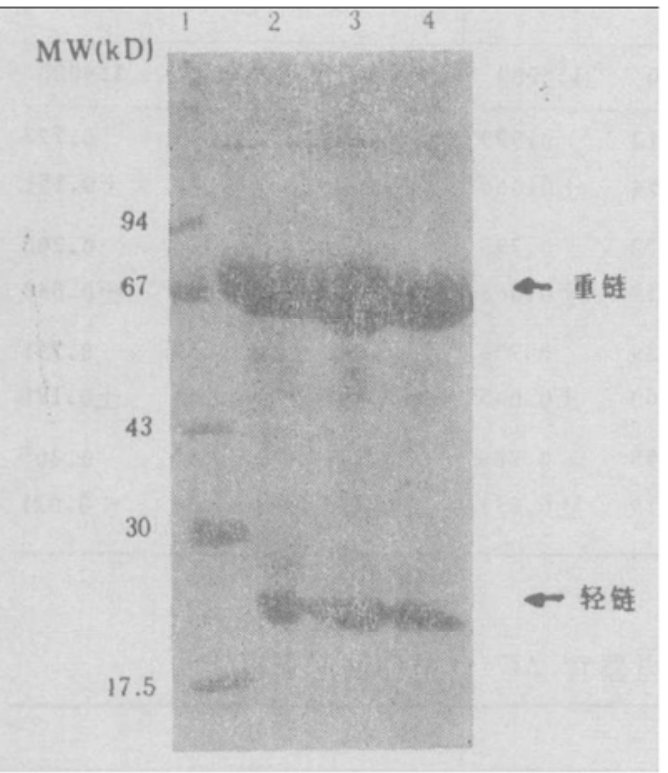

图 1 纯化的抗人脑 $\mathrm{AChE}$ 单克隆抗 体的 SDS-PAGE

1一标准分子畫勇白, 2- $\mathrm{E}_{11}, 3$ $\mathrm{H}_{11}, 4-\mathrm{B}_{10}$

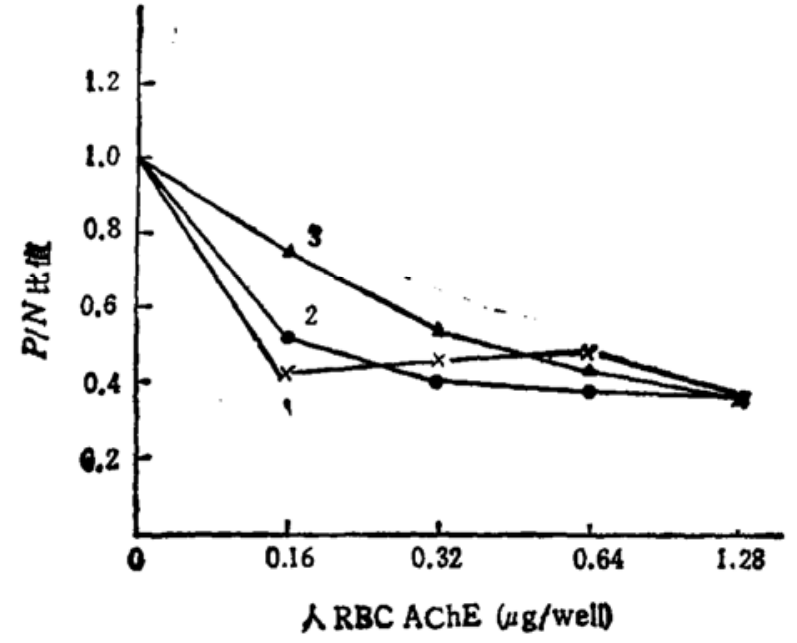

图 2 抗人脑 $\mathrm{AChE}$ 单克隆抗体与不同量人红细胸膜 $A C b E$ 的抗原抗体反应(BL ISA)P $/ N$ 比值

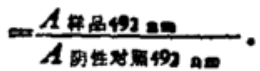

纣体稀释度: $B_{10}$ 及 $\mathrm{H}_{11}, 1: 2000 ; \mathrm{E}_{11}, 1: 1000$. $1-\mathrm{B}_{260} 2-\mathrm{H}_{13}, 3-\mathrm{E}_{11}$

\section{2 抗人脑 $\mathrm{AChE}$ 单克隆抗体的交叉免落反应性}

ELISA 检测时,每孔包被人脑、人红细胞膜或电鳐电器官 $A \mathrm{ChE} 0.16 \mu \mathrm{g}$, 阴性对照组以 包被缓冲液代替抗原. 从表 1 中看出, $B_{10}, H_{11}$ 及 $E_{11}$ 三个单克隆抗体与电鳐 AChE 有明显的交叉反应, 吸光值对抗 体浓度呈现明显的依赖性; 而与人红细 胞膜 AChE 反应的消光值与无抗 原 对 照组相比无明显差异. 增加人红细胞膜 $\mathrm{AChE}$ 的包被量 (从每孔 $0.16 \mu \mathrm{g}$ 增至 $1.28 \mu \mathrm{g})$ 时, $\mathrm{B}_{10}, \mathrm{H}_{11}$ 及 $\mathrm{E}_{11}$ 三个单克 隆抗体与人红细胞膜 $\mathrm{AChE}$ 反应的吸 光值并不相应地增加, 反而下降, 低于 无抗原阴性对照组 (图 2). 表明三个抗 人脑 $\mathrm{AChE}$ 单克隆抗体与人红细胞 膜 AChE 之间无交叉免疫反应性.

\section{3 抗人脑 $\mathrm{AChE}$ 单克隆抗体所针对} 的抗原决定穊的性质

分别以 $\mathrm{B}_{10}, \mathrm{H}_{11}$ 及 $\mathrm{E}_{11}$ 单克隆抗体 与天然人脑 AChE ELISA 反应的消光

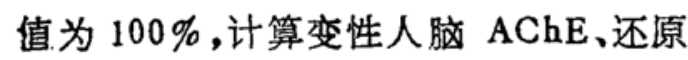
并烷化的电鳐电器官 $\mathrm{AChE}$ 及无抗原

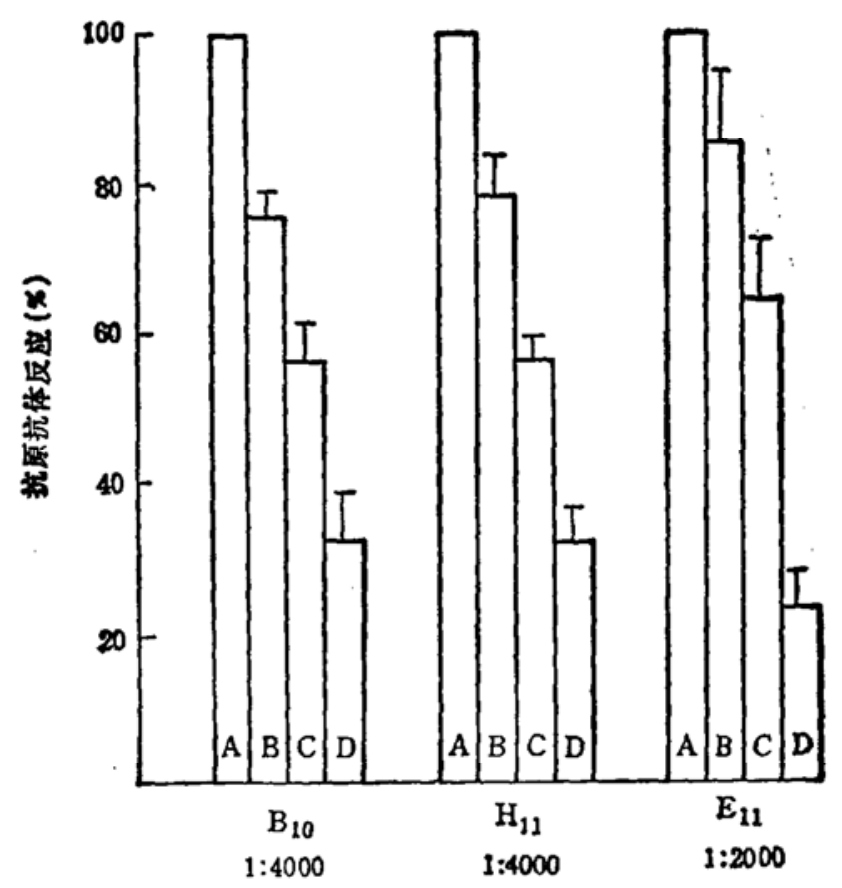

图 3 抗人脑 $\mathrm{AChE}$ 单克隆抗体与变性人脑 $\mathrm{AChE}$ 及变性 电觘电器官 $\mathrm{AChE}$ 的抗原抗体反应(BLISA) $\mathrm{A}$ 一天然人䏽 $\mathrm{AChE}, \mathrm{B}$ 一一变性人脑 $\mathrm{AChE}$, $\mathrm{C}$ 一一变性电解电器官 $\mathrm{AChE}, \mathrm{D}$ 一一无抗原对照 对炤组的百分比. 可以看出, 三个单克隆抗体与变性人脑 AChE 及还原并烷化的电鳐电器 官 
表 1 抗人脑 $\mathrm{AChE}$ 单克隆莯体同人 $\mathrm{RBC} A \mathrm{ChE}$ 及电䁘 $\mathrm{AChE}$ 的免度交叉反应"

\begin{tabular}{|c|c|c|c|c|c|c|c|c|c|}
\hline \multirow{2}{*}{$\begin{array}{l}\text { 抗 作 } \\
\text { 稀 释 空 }\end{array}$} & \multicolumn{3}{|c|}{ B. } & \multicolumn{3}{|c|}{$H_{11}$} & \multicolumn{3}{|c|}{$E_{11}$} \\
\hline & $1: 2000$ & $1: 4000$ & 1:Sv00 & $1: 20.00$ & $1: 4000$ & $1: 8000$ & $1: 1000$ & $1: 2090$ & $1: 4000$ \\
\hline 人 脑 & 2.125 & 1.828 & 1.385 & 2.222 & 1.512 & 0.999 & 1.465 & 1.175 & 0.778 \\
\hline $\mathrm{AChE}$ & \pm 0.279 & $\pm 0.0+2$ & \pm 0.106 & \pm 0.104 & \pm 0.054 & \pm 0.066 & \pm 0.062 & \pm 0.141 & \pm 0.151 \\
\hline 人 $\mathrm{RBC}$ & 0.4132 & 0.462 & 0.330 & 0.426 & 0.372 & 0.293 & 0.259 & 0.246 & 0.200 \\
\hline $\mathrm{AChE}$ & \pm 0.036 & \pm 0.102 & \pm 0.030 & \pm 0.033 & \pm 0.059 & \pm 0.063 & \pm 0.073 & \pm 0.089 & \pm 0.088 \\
\hline 电 媱 & 2.040 & 1.641 & 1.324 & $1.9: 9$ & 1.329 & 0.906 & 1.308 & 1.039 & 0.751 \\
\hline $\mathrm{AChE}$ & \pm 0.083 & \pm 0.060 & \pm 0.102 & \pm 0.077 & \pm 0.060 & \pm 0.045 & \pm 0.109 & \pm 0.144 & \pm 0.176 \\
\hline 无抗原 & 0.494 & 0.533 & 0.431 & 0.49 .4 & 0.355 & 0.289 & 0.221 & 0.230 & 0.205 \\
\hline 阴性刘照 & \pm 0.233 & \pm 0.231 & \pm 0.192 & \pm 0.213 & \pm 0.019 & \pm 0.031 & \pm 0.113 & $\pm 0.03 x$ & \pm 0.021 \\
\hline
\end{tabular}

a) $A_{4: n: 0}, n=4, \bar{M} \pm S D$.

表 2 高碘酸样化处理对还原烷學化的电鳐电器官 ACh E抗原性的影响e)

\begin{tabular}{|c|c|c|c|c|}
\hline 抗 体 & $B_{10}$ & $\mathrm{H}_{11}$ & $E_{11}$ & 阴性对炤 \\
\hline 稆释度 & $1: 8000$ & $1: 8000$ & $1: 4000$ & \\
\hline 嵫化处理的 & 0.444 & 0.517 & $0.4: 6$ & 0.097 \\
\hline ACbi: & \pm 0.031 & $\pm 0.0=3$ & \pm 0.005 & $\pm^{13.0436}$ \\
\hline 正 常 & 0.414 & 0.398 & 0.436 & 0.116 \\
\hline $\mathrm{AChE}$ & \pm 0.014 & \pm 0.015 & \pm 0.041 & $\pm 0.01 t$ \\
\hline
\end{tabular}

a) $A_{4, i \mathrm{~m}}, n=3, \bar{M} \pm \mathrm{SD}$.

AChE 仍有抗原抗体反应,但其反应性明显低于天然的人脑 AChE 抗原(图 3). 说明这三个 抗人脑 AChE 单克隆抗体所针对的抗原决定簇是连续性顺序决定簇。从表 2 中看出,高碘酸 笔化处理前后还原并烷化的电鳐 $\mathrm{AChE}$ 与抗人脑 $\mathrm{AChE}$ 单克隆抗体反应性无明显差异,表明 抗人脑 $\mathrm{AChE}$ 单克隆抗体 $\mathrm{B}_{10}, \mathrm{H}_{11}$ 及 $\mathrm{E}_{11}$ 所针对的抗原决定簇可能不是 $\mathrm{AChE}$ 的糖基侧链, 而是其多肤序列。

\section{4 抗人脑 AChE 单克隆抗体对酶活性的影响}

将纯化的人脑 $\mathrm{AChE}$ 按 1:50(V/V) 以 $0.1 \mathrm{~mol} / \mathrm{L}$ 磷酸钠缓冲液 ( $\mathrm{pH} 7.4)-0.1 \%$ Triton X-100 稀释后,取 $16 \mu 1$ 与不同量的纯化的抗人脑 AChE 单克隆抗体反应, 观察其对 AChE 活性的影响. 当 $\mathrm{B}_{10}, \mathrm{H}_{11}$ 及 $\mathrm{E}_{11}$ 三个单克隆抗体每管加量为 15 及 $30 \mu \mathrm{g}$ 时, 抗体与 $\mathrm{AChE}$ 的摩尔比值分别约为 43:1 及 86:1 (AChE 单体分子量依 $66 \mathrm{kD}$ 计算, 单克隆抗体单体 分子 量依 $180 \mathrm{kD}$ 计算), 对人脑 $\mathrm{AChE}$ 活性无明显影响. 表明三个单克隆抗体在 $\mathrm{AChE}$ 分子上 的结合部位位于距酶活力中心很远的地方.

\section{3 讨 论}

人脑及人红细胞膜 $\mathrm{AChE}$ 一级结构的全序列尚无报道. 现有文献报道的抗人红细胞膜 或抗人脑 $\mathrm{AChE}$ 单克隆抗体 ${ }^{[2.31}$ 多数对二者没有选择性, 无法判断二者一级结构的差异性. $B_{10}, H_{11}$ 及 $E_{11}$ 三个单克隆抗体能与变性的人脑 AChE 及还原并烷化的电鳐 AChE 发生抗 原抗体反应, 说明作用于 $\mathrm{AChE}$ 分子的顺序决定簇. 高碘酸能选择性地使糖分子中的连二 羟基或连三羟基处断裂,使蛋白分子上的糖基侧链破坏. 经过高碏酸处理的电鳐 $\mathrm{AChE}$ 抗原 
生不消失, 又说明这三个单克隆抗体可能作用于 $\mathrm{AChE}$ 的非糖基顺序决定簇. 由此可以推论 三个单克隆抗体作用的人脑 $\mathrm{AChE}$ 分子上的抗原决定簇的一级结构与电鳐电器官 $\mathrm{AChE}$ 相 似, 而 $\mathrm{B}_{10}, \mathrm{H}_{11}$ 及 $\mathrm{E}_{11}$ 与人红细胞膜 $\mathrm{AChE}$ 无交叉免疫反应性意味着这三个单克隆抗体所针 对的抗原决定簇不存在于人红细胞膜 $\mathrm{AChE}$ 分子上. 三个单克隆抗体对变性人脑 $\mathrm{AChE}$ 及 还原并烷化电鳐 $\mathrm{AChE}$ 的反应性低于天然 $\mathrm{AChE}$, 似说明抗原抗体反应仍有一定程度的构象 依赖性. 抗原决定簇分为顺序决定笶及构象决定簇是相对的, 可能顺序决定簇与抗体的反应 依赖其基团结构与抗体结合部位的吻合, 而构象决定筷是由一级结构决定的三级结构形成的. $B_{10}$ 及 $\mathrm{H}_{11}$ 与人脑 $\mathrm{AChE}$ 有较强的结合力, 用 ELISA 法测定其抗体反应最高值在相同条件 下约为抗人红细胞膜单克隆抗体 $4 \mathrm{~F}_{19}{ }^{\left[{ }^{[2]}\right.}$ 及抗电鳐 $\mathrm{AChE}$ 单克隆抗体 $2 \mathrm{G}_{\mathrm{x}}{ }^{[4]}$ 的 8 倍, 为抗电鳐 AChE 单克隆抗体 $1 \mathrm{H}_{11}{ }^{\left[{ }^{4]}\right.}$ 的 10 倍, 可望用于人脑及其它神经组织 AChE 的免度组化及代谢 研究, 以及先天性神经管缺损的产前诊断. 由于这三个抗体能识别变性 AChE, 弥补了 AChE 活性测定法对变性 AChE 无法测定的不足.

\section{參 考堿}

[1] Younkin, S. G., Goodridge, B., Katz, J. et al., Fed. Proc., 1986, 45: 2982.

[2] Rasmussen, A. G., Sorensen, K., Selmer, J. et al., Clin. Chim. Acte, 1987, 166: 17.

[3] Rakonczay, Z., Brimijoin, S., Cell Mol. Neurobiol., 1988, 8: 85.

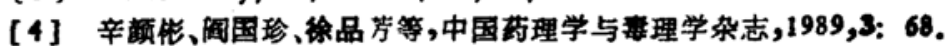

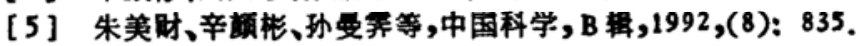

[6] Ott, P., Jenny, B., Brodbeck, U., Ewr. J. Biochem., 1975, 57, 469.

[7] 功曼手、高天栋、邢志勇等,生物化学杂志,1985,1: 47.

[8] Woodard, M. P., J. Immwnol. Methods, 1985, 78: 143.

[9] Parham, P., Androlewicz, M. J.. Brodsky, F. M. et al., J. Immunol. Methods, 1982, 53 :133.

[10] 本风珍、孙曼节,军事医学科学院院刊, 1986,10: 211 . 